\title{
Graphing the Complex Roots of Quadratic Functions on a Three Dimensional Coordinate Space
}

\author{
Aravind Narayan
}

\begin{abstract}
We are familiar with the geometric definition of Real roots of a Quadratic function as the $x$-intercept of the Quadratic functions graph; however such a geometric definition is not given for the Complex roots of a Quadratic function. This paper geometrically defines complex numbers as solution of Quadratic Equations. Inorder to achieve this a new coordinate space is defined where given a quadratic function with complex solution we can geometrically plot complex solutions to it, in this new coordinate frame. Thus the solution to any Quadratic equation with complex solutions can be derived geometrically. Also this coordinate frame will define the entire Complex Plane as a Solution Space of Quadratic functions with complex roots.

The paper further provides ideas and conjures to extend the research into polynomials of degree higher than two i.e. the cubic polynomials and higher. The author hopes that further research could develop the method to geometrically derive complex solutions to cubic and higher degree polynomials, and the complex numbers will no more be fictitious quantities but quantities with rigorous geometric definitions.
\end{abstract}

\section{Introduction}

The Complex number arose along with trying to find roots of quadratic and higher degree polynomial Equations. This required going out of the real numbers for a solution, which gave rise to a mathematical entity we now know as the imaginary number. The imaginary number ' $i$ ' represents the square root of a negative ' 1 '.

Square root of negative numbers was considered as early as 1st Century. However the first major work using Complex numbers occurred in the 1500's as solution of a Quadratic Equation. However mathematicians did not readily accept the complex numbers. It took centuries to convince mathematicians to accept complex numbers. The reason for this could be the lack of a geometric definition of complex numbers as roots of Quadratic functions, while the real roots have a geometric definition.

In 1600's Rene Descartes developed the Cartesian Geometry, establishing a connection between algebra and geometry. In Descartes time it was common to translate between the algebraic and geometric form of polynomial equations. When the polynomial functions was plotted geometrically only the real solutions gave a geometric meaning. This must have led Descartes to call the complex solutions as false and the square root of the negative number as imaginary.

The Quadratic Function when geometrically plotted on Cartesian coordinate system the roots of a Quadratic function is defined as the points where the function's plot intersects the X-axis. When the Quadratic equation of the function is solved the X-intercept values are the solution of the Quadratic Equation. However some Quadratic equations do not give real solutions. Instead the solutions contain square root of negative quantities. Algebraic representation of this solution is defined considering the square root of negative quantities as imaginary number ' $i$ '. Geometrically these are the Quadratic functions whose plots never intersect the X-axis. (Reference; Appendix C)

Had the complex number been defined as a geometric solution to the polynomial equation or for that matter at least for Quadratic function mathematicians wouldn't have found it hard to accept this imaginary number. If such was the case the resistance to the complex solution would not have been an issue and it would have been accepted on a firm geometric foundation.

\section{Definitions}

Defining the Quadratic function with Complex roots as 'Complex Quadratic Function'

Most of the Quadratic Equations have real solution. In-fact it is only a special class of functions which will give Complex solutions for its Quadratic Equations. Let us define this class of Quadratic Functions having only complex roots as the 'Complex Quadratic Function'.

We will define the Complex Quadratic Function as the Quadratic Functions of the form

$$
\pm\left(A x^{2} \pm B x+C\right)=f(x)
$$

The required conditions for a Quadratic Equation to be a Complex Quadratic Function is

a) The coefficients ' $A$ ' \& ' $C$ ' should have the same sign

b) And $-2 \sqrt{A C}<B<+2 \sqrt{A C}$ 
Any Quadratic Function that does not meet the above conditions will have real roots i.e. the plot of the Quadratic function will intersect the X-axis.

Appendix $C$ gives a graphical view of the form of quadratic functions with complex roots

\section{Standard-Complex Quadratic Function}

Now we will define a standard form for the 'Complex Quadratic Function'. A Quadratic Function is defined to be a 'Standard Complex Quadratic Function' if the coefficient of, ' $\mathrm{x}$ ' is 1 ' i.e. ' $\mathrm{A}=1$ '

Given a 'Complex Quadratic Function' of the form

$$
\left(A x^{2} \pm B x+C\right)=f(x)
$$

We first write it in the Quadratic Equation form as

$$
\left(A x^{2} \pm B x+C\right)=0
$$

We can reduce it to the Standard Complex Quadratic Equation (SCQE) by dividing throughout by 'A'

Let us name the constants

$$
\left(\frac{A}{A} x^{2} \pm \frac{B}{A} x+\frac{C}{A}\right)=0
$$

And

$$
\frac{\mathrm{B}}{\mathrm{A}}=\mathrm{b}
$$

$$
\frac{C}{A}=c
$$

The Standard- Complex Quadratic Equation can then be written as

$$
\left(x^{2} \pm b x+c\right)=0
$$

Given a Complex Quadratic Function of the form

$$
-\left(A x^{2} \pm B x+C\right)=f(x)
$$

We first write it in the Quadratic Equation form as

$$
-\left(A x^{2} \pm B x+C\right)=0
$$

We can reduce it to the Standard Complex Quadratic Equation (SCQE) by dividing throughout by '-A'

$$
\left(\frac{-A}{-A} x^{2} \pm \frac{B}{-A} x+\frac{-C}{-A}\right)=0
$$

To bring it to the form

Equivalent to

$$
\begin{aligned}
& \left(x^{2} \mp b x+c\right)=0 \\
& \left(x^{2} \pm b x+c\right)=0
\end{aligned}
$$

Thus we define our 'Standard Complex Quadratic Equation' as

$$
\left(x^{2} \pm b x+c\right)=0
$$

Definition

Thus we define the 'Standard Complex Quadratic Function' as Quadratic functions of the form

Such that ' $b$ ' has the following limits

$$
\left(x^{2} \pm b x+c\right)=f(x)
$$

$-2 \sqrt{c}<b<+2 \sqrt{c}$ 


\section{Complex Quadratic Constant}

We will also name the constant 'c' of our 'Standard Complex Quadratic Equation' as the 'Complex Quadratic Constant'.

If we plot the 'Standard Complex Quadratic Function' in the ' $\mathrm{X}-\mathrm{Y}$ ' plane we will have the ' $\mathrm{Y}$ '- intercept to be equal to the 'Complex Quadratic Constant' ' $c$ '.

\section{Critical Quadratic Constant}

We will also define an additional constant given as

$$
\mathrm{k}=-\left(\frac{ \pm b}{2}\right)
$$

If we plot the 'Standard Complex Quadratic Function' in the X-Y plane the Quadratic curve will have a critical point. ' $k$ ' Gives the X-coordinate value of critical point of the 'Quadratic Function'

This critical point can be derived using the calculus as

$$
\begin{gathered}
\frac{d}{d x}\left(x^{2} \pm b x+c\right)=0 \\
(2 x \pm b x)=0 \\
\mathrm{x}=-\left(\frac{ \pm b}{2}\right)
\end{gathered}
$$

Note here that this Critical Quadratic Constant is the Real part of the complex solution of the 'Complex Quadratic Equation'

\section{The Complex roots of Complex Quadratic Equation}

We have seen that we can bring any Complex Quadratic Function to its Standard-Complex Quadratic Function' form and the complex roots of the Complex Quadratic Function is equal to the complex roots of its corresponding Standard-Complex Quadratic Function.

Thus in-order to define our geometric coordinate space of complex solutions it is enough if we will analyze the solution set of Standard-Complex Quadratic Functions.

Given any complex number and its conjugate, this pair of complex numbers will be the roots of a unique Standard-Complex Quadratic Function

"Thus we can state that the Set of complex numbers is the solution space of the roots of Standard-Complex Quadratic Functions”

Fact: A set of Standard-Complex Quadratic Functions which is defined by a particular Complex Quadratic Constant ' $c$ ' will have its Roots lying along a circular contour in the complex plane with radius ' $\sqrt{c}$ '

Approach

\section{Defining the Complex Coordinate Space}

For a Quadratic function with a Complex roots (Complex solutions for its QE) the geometric plot of the Quadratic function does not intersect the X-axis. From the graphical plot we have visual evidence that the parabola will never meet the $\mathrm{X}$-axis in the Coordinate system. We can thus establish that there is no solution in the two dimensional space where our Quadratic function with complex roots exists.

So in-order to define our solution we have to move to a higher dimension. This is our basic approach. We will conceptualize our Quadratic function to be existing in a three dimensional space. The X-Y plane is now only an element of the 3D space. Also from our algebraic experience with the roots of a complex Quadratic function we know that we need to extend our number system to include square root of negative numbers as is evident from the algebraic solutions. Thus we need two additional definitions, 'A Third dimension' and 'An extension of the number system to square root of negative quantities', i.e. imaginary number ' $i$ '

Thus our approach here is to extend our two dimensional coordinate system to define a new Coordinate system where we can plot complex roots as geometric solutions of Quadratic Functions just as we do for Real roots in two dimensional case.

\section{Defining the Co-ordinate System}

Thus we will extend our two dimensional space to a third dimension ' $Z$ ' with ' $Z$ ' having square root of negative quantities i.e. Square, root of negative numbers denoted ' $i$ '.

So now we have a three dimensional geometric coordinate space, however our geometric coordinate system is not yet complete. We will define an additional function between the $\mathrm{Y}$-axis and the imaginary ' $\mathrm{Z}$ ' axis. This function will define a parabola in the 'Y-Z' plane of our three dimensional space. This parabola in the Y-Z plane 
will be a fourth coordinate (this will be a tertiary coordinate- the $\mathrm{X}, \mathrm{Y} \& \mathrm{Z}$ axes are our primary coordinates) in our geometric space.

The function defining the parabolic coordinate of our geometric space is,

$$
y=\left|(z i)^{2}\right|
$$

The parabola is symmetric to the positive Y-axis. This fourth coordinate is a critical coordinate in defining the Complex Solution of our Quadratic Equation i.e. geometrically plotting the complex Roots of our Geometric Function.

Let us call this parabola as the 'Complex Parabolic Coordinate'

Both the $\mathrm{X} \& \mathrm{Y}$ axes are real axis \&

The $\mathrm{Z}$ axis is the imaginary axis.

In this plot however our complex plane would be the $\mathrm{X}-\mathrm{Z}$ plane. The complex solutions of the Complex Quadratic Equations are plotted in the X-Z plane. The X-Y Plane forms the Real Plane and we have only Real values in this plane.

\section{The Complex Parabolic Coordinate System}

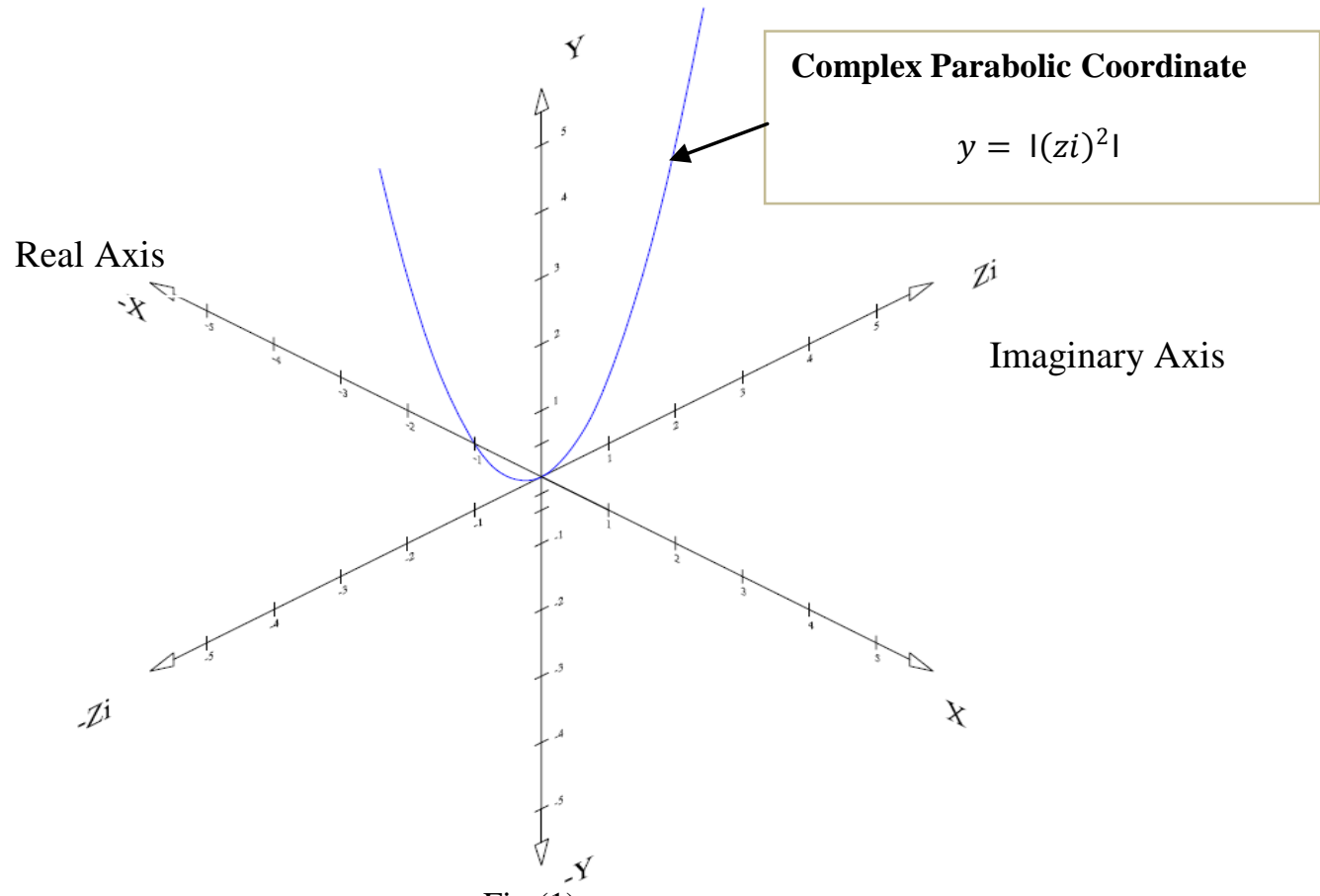

Fig (1)

\section{The Graphing Procedure for Determining the Complex Roots of Quadratic Function}

Here we define the step by step procedure for geometrically plotting the roots of a given quadratic function.

1. Given a Quadratic function identify if it is a 'Complex Quadratic Function'

2. If so then reduce the function to its 'Standard Complex Quadratic Function' form by dividing throughout by the coefficient of ' $\mathrm{x}^{2}$ '. Plot this function in the X-Y Real plane.

3. Define the Complex Quadratic Constant of the Standard Complex Quadratic Function. This is the 'Yintercept' of the graph of 'Standard Complex Quadratic Function' in the X-Y Plane.

4. Identify the Complex Quadratic Constant on the Y-axis which is the Y-intercept of 'Standard Complex Quadratic Function'. Draw a line through this Y-intercept perpendicular to the Y-axis and parallel to the imaginary 'Z-axis' (i.e. on the Y-Z plane) to intersect the 'Complex Parabolic Coordinate'. Identify the intersection points on the 'Complex Parabolic Coordinate'. From these points drop perpendiculars to the imaginary Z-axis. This will give us our corresponding imaginary points ' $\pm z i$ ' 
5. We now draw a circle in the $X-Z$ plane with the Center at the origin and passing through our imaginary point ' $+z i$ ' $\&$ ' $-z i$, identified on the Z-axis. This circle will have a radius equal to the square root of the Complex Quadratic Constant

The complex root of our Complex Quadratic Function will lie on this Circular Contour. In fact the set of all complex solutions of all the Complex Quadratic Functions which has a Complex Quadratic Constant equal to ' $c$ ' will lie on this circular contour. Thus this circular contour represents the solution set for all Complex Quadratic Functions with Complex Quadratic Constant equal to a particular ' c'.

6. Now we identify the critical point (minima) of our Standard Complex Quadratic Function. We then determine the 'X-coordinate' value of this critical point by dropping a perpendicular to the $\mathrm{X}$-axis from this point. Algebraically this ' $X$-coordinate' value is equal to $\left[-\left(\frac{b}{2}\right)\right]$, where ' $b$ ' is the coefficient of ' $x$ ' in the Standard Complex Quadratic Function of our given function.

7. From this 'X-coordinate' intercept (value) we draw a line perpendicular to the $\mathrm{X}$-axis and on the $\mathrm{X}-\mathrm{Z}$ plane to intersect our circular contour defined in Step 5. This perpendicular intersects the Circle at two unique points lying on the complex ' $\mathrm{X}-\mathrm{Z}$ ' plane. These two points are the complex solution of the given Complex Quadratic Function.

Note: each point on the complex plane represents a Complex number.

'Thus we are able to geometrically derive the Complex solution of a Quadratic function'

Solving the Complex Quadratic Function in this Coordinate Frame

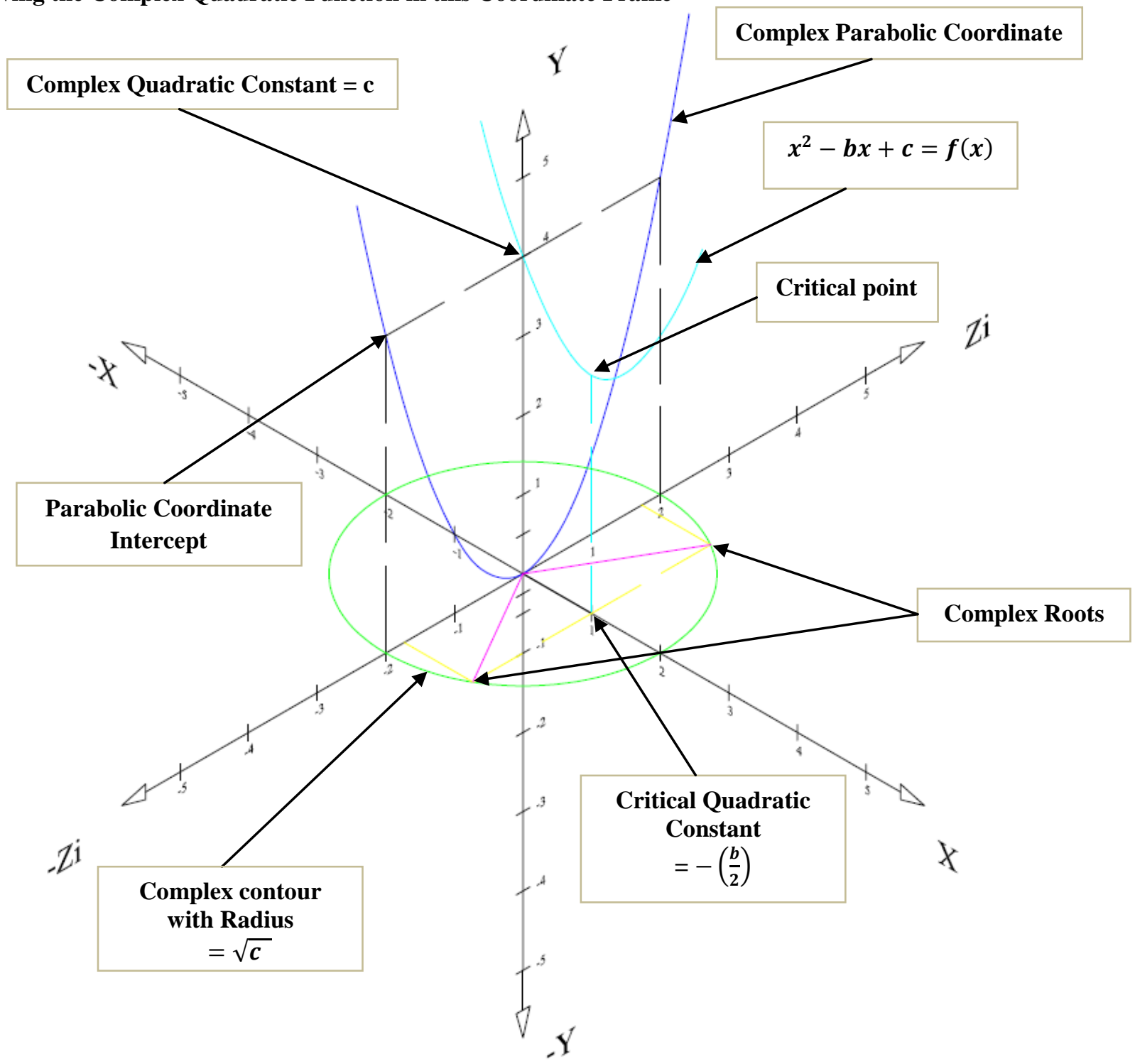

Fig (2) 
A detailed view of each Coordinate plane is provided in Appendix B: The Axial Planes of Our Coordinate System

\title{
The Critical Points
}

\section{Conclusions \&Conjures}

The Critical points of a polynomial functions plays a major role in defining the roots of the function. For a quadratic function with real roots the real roots are equidistant from the critical point. Also the real roots are equidistant from the X-coordinate point of the Critical point. This is true for the complex roots as well. When the complex roots are plotted geometrically the complex roots lie on line passing through the $\mathrm{X}$ coordinate of the critical point perpendicular to the 'X-axis' and parallel to the imaginary 'Z-axis'.

A logical analysis of the roots of a polynomial function leads to the following

$>$ Any polynomial function of odd degree will have one Real root.

$>$ A polynomial function will have complex roots having real part equal to the X-coordinate of Critical points

\section{Critical Points Defining Complex Roots}

We have seen that the defined Complex roots are determined by the critical points. However all the critical points do not generate complex roots. The conditions for a Critical point to give complex roots depend on the distribution of critical points. We will define this on a case by case-basis.

Case-A; the Critical Point in an Interior critical point i.e. it is preceded and succeeded by another Critical Point

If the graphical plot of the polynomial function has three consecutive critical points on the same side of the $\mathrm{X}$-axis i.e. above or below the $\mathrm{X}$-axis then the critical point in the center will give a Complex root. This complex root will have real part equal to the $\mathrm{X}$-coordinate of this critical point.

Case- $B$; The Critical point is a terminal one i.e. there is no, preceding or succeeding critical point. Here we have two different Situations

$>$ If the critical point is the first critical point that is there is no preceding critical point it will define complex solution if the next critical point (the succeeding one) lies on the same side of $\mathrm{X}$-axis as this critical point and the opening plot from the critical point move away from the $\mathrm{X}$-axis and never intersects the $\mathrm{X}$-axis.

$>$ If the critical point is the last critical point that is there is no succeeding critical point it will define complex solution if the preceding critical point (the previous one) lies on the same side of X-axis as this critical point and the opening plot from the critical point move away from the $\mathrm{X}$-axis and never intersects the $\mathrm{X}$-axis.

"Opening plot means if the Critical point is a terminal one i.e. the first or last one then one side of the critical point does not lead to another critical point but extends infinitely in a particular direction"

A critical point is defined in the same sense as is used in Calculus.

With the above analysis we can safely give the following conjure

Conjure

"For a polynomial function with Complex roots all the complex roots will lie on a line on the X-Z complex plane perpendicular to the $X$-axis and passing through the $X$-coordinate of critical points, however all critical points do not generate complex roots"

\section{Acknowledgement}

The Author acknowledges and thanks the use of online Quadratic Graphing calculator by 'Math Portal.org' http://mathportal.org/calculators/quadratic-equation/quadratic-function-grapher.php

\author{
References \\ Wikipedia Articles: Complex numbers \\ Wikipedia Articles: Complex plane \\ Wikipedia Articles: Quadratic function \\ Wikipedia Articles: Quadratic equation \\ Paul Dawkins: Calculus I (Link: http://tutorial.math.lamar.edu/)
}

\section{APPENDICES}

Appendix A: Geometric Solution of a Complex Quadratic Function, an Example

Appendix B: The Axial Planes of our Coordinate System

Appendix C: The Complex Quadratic Equations 


\section{Appendix A: Geometric Solution of a Complex Quadratic Function, an Example}

Q) Solve the following Quadratic Function geometrically

\section{Solution}

$$
f(x)=x^{2}-2 x+4
$$

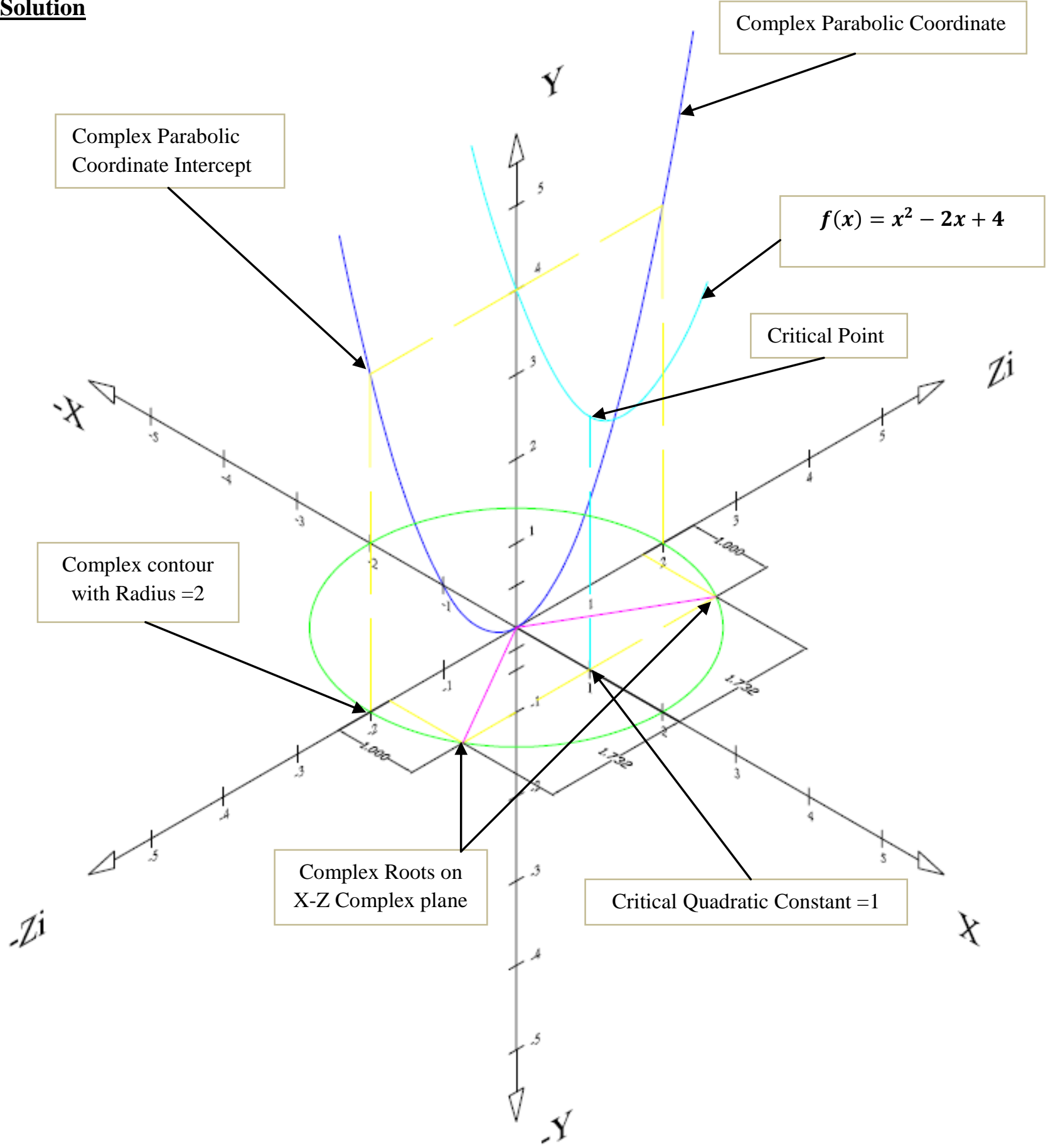

Fig (3)

Complex Quadratic Constant ' $\mathrm{c}$ ' $=4$

Radius of the Complex Contour $\sqrt{c}=\sqrt{4}=2$

Critical Quadratic Constant $=-\left(\frac{-b}{2}\right)=-\left(\frac{-2}{2}\right)=1$

From the plot on the complex $X-Z$ plane we derive the solution as

Complex Solution $=(1 \pm 1.732 i)$ 
This is the complex solution for any Quadratic Equation which can be reduced to the above 'Standard Complex Quadratic Function' form e.g.

$$
\begin{gathered}
f(x)=2 x^{2}-4 x+8 \\
f(x)=3 x^{2}-6 x+12 \\
f(x)=4 x^{2}-8 x+16 \\
f(x)=5 x^{2}-10 x+20 \\
f(x)=-2 x^{2}+4 x-8 \\
f(x)=-3 x^{2}+6 x-12 \\
f(x)=-4 x^{2}+8 x-16 \\
f(x)=-5 x^{2}+10 x-20
\end{gathered}
$$

Etc.....

\section{Appendix B: The Axial Planes of our Coordinate System}

\section{The X-Y Real Plane}

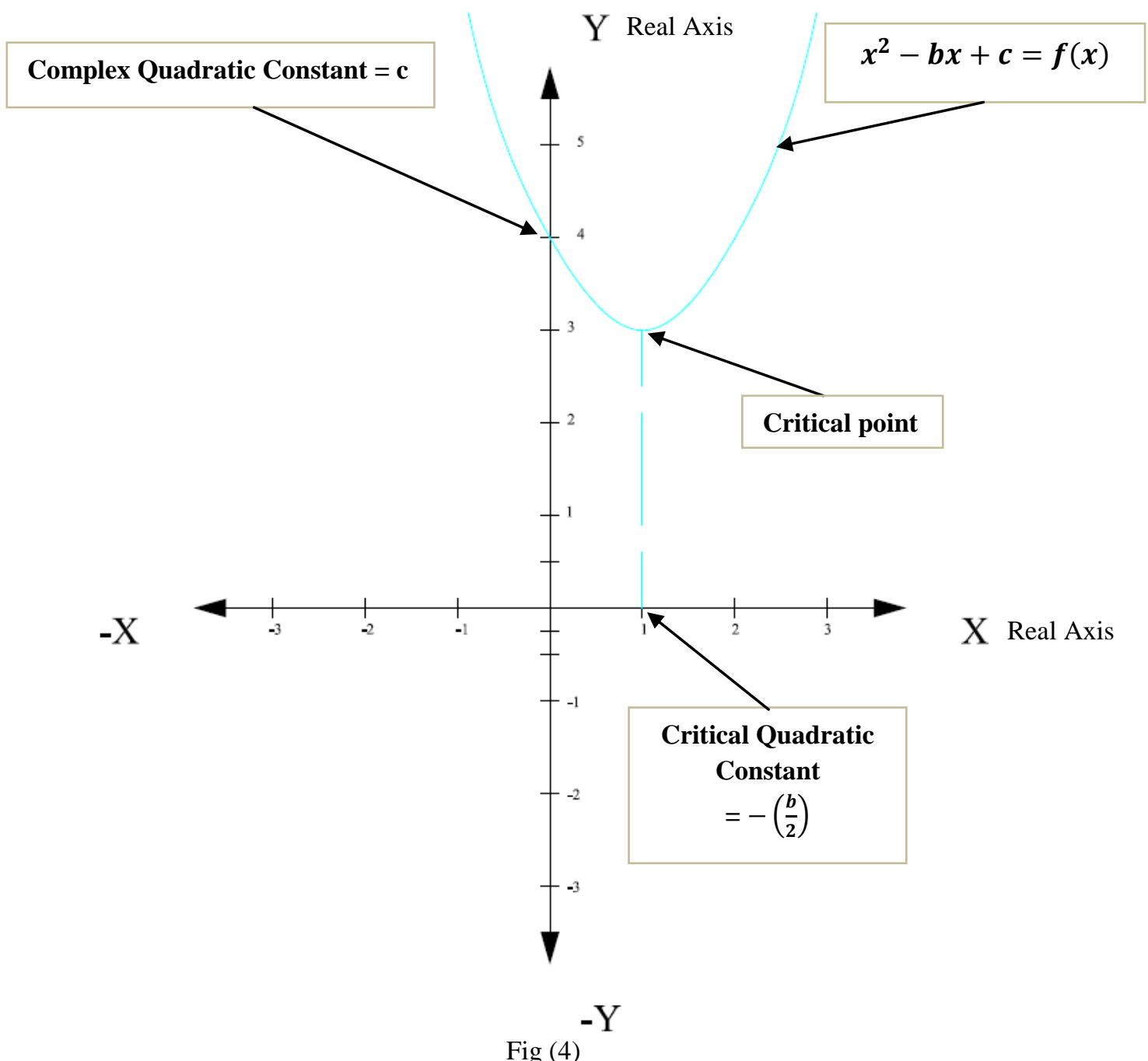

Plot of the function in Standard Complex Quadratic Function in the 'X-Y' Real Plane, with the Y-intercept being the Complex Quadratic Constant 
The Y-Z Coordinate Plane (with the Complex Parabolic Coordinate)

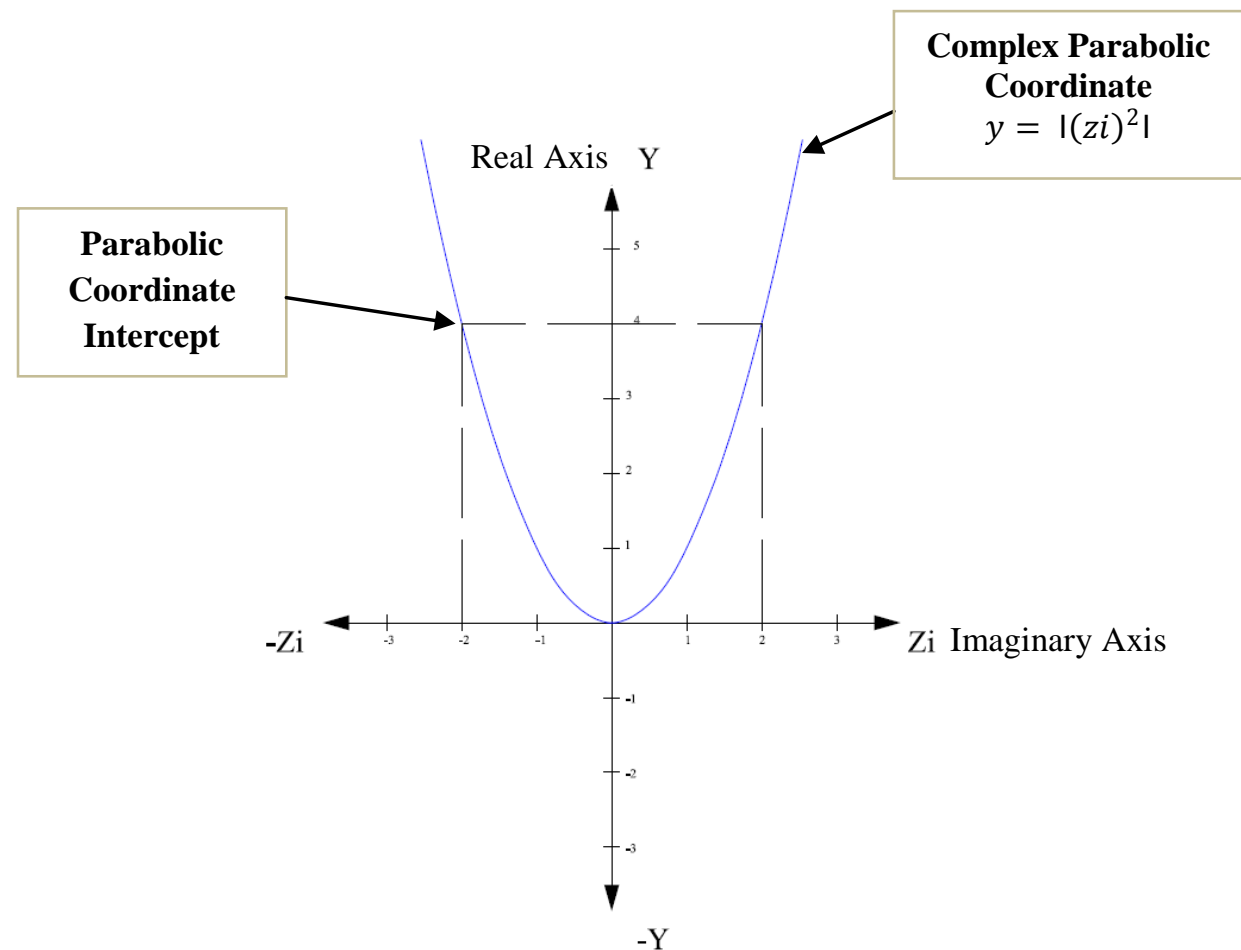

Fig (5)

Plot of the Y-Z-Complex Parabolic coordinate. Each point on the Real Y-Axis is plotted to two points on the imaginary 'Z-axis'. These two points are conjugates of each other and defines a Circular Contour on the Complex plane.

The X-Z Complex Plane- The Solution Plane

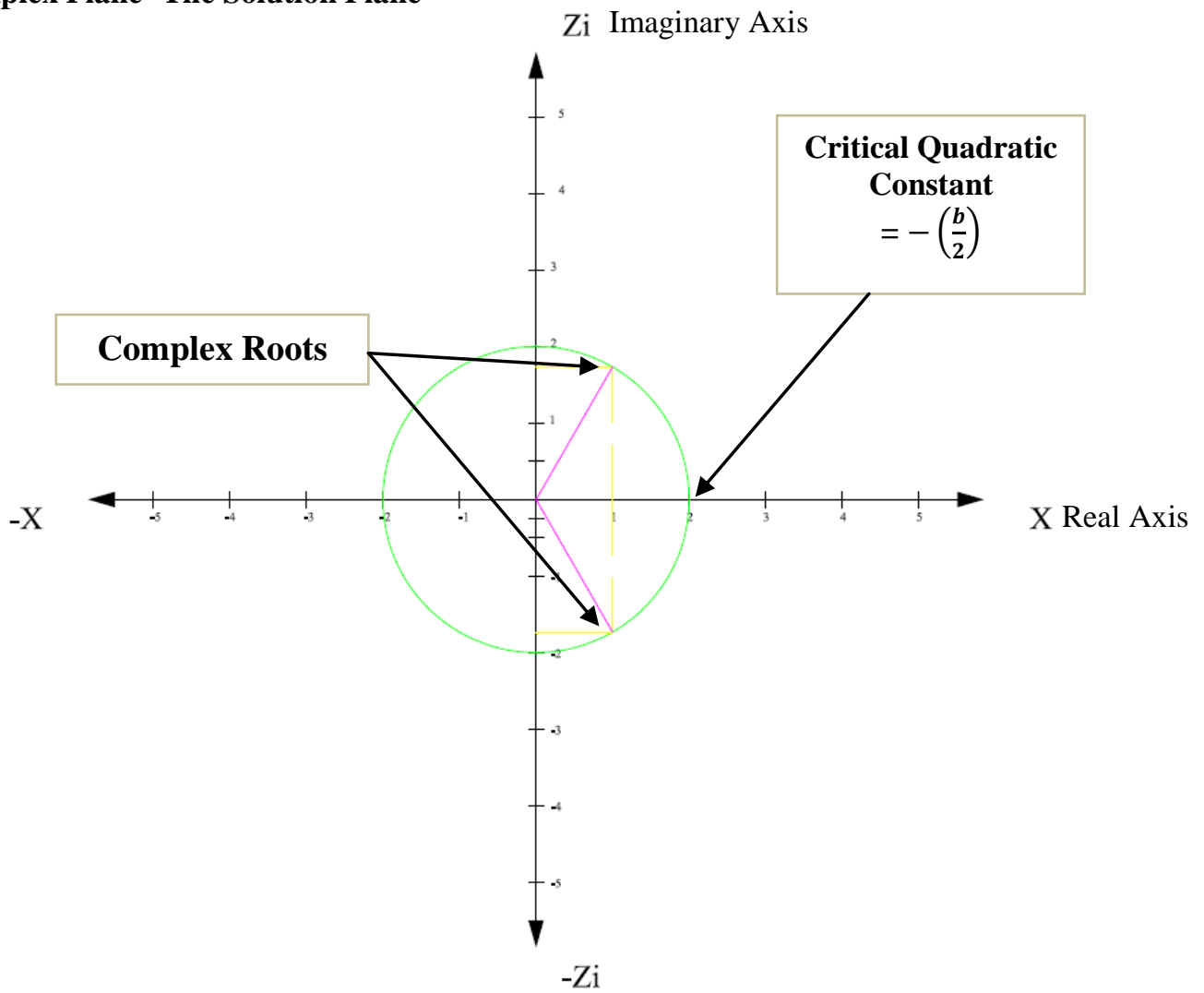

Fig (6) 
This plane is the ' $\mathrm{X}-\mathrm{Z}$ ' - Complex plane, which is also the solution plane, where the complex solutions are plotted. Each Point on the Real 'Y- Axis' will define a circular Contour in this Plane through the Complex Parabolic Coordinate. All the Quadratic Equation with complex solution lying on this Circular contour will have its Standard Complex Quadratic Function's plot pass through the Y-coordinate defining the Complex contour

\section{Appendix C: The Complex Quadratic Functions}

There is only a certain class of Quadratic Functions that give complex solutions. This class of functions is defined as the Complex Quadratic Functions meeting the following conditions

$$
\begin{gathered}
\pm\left(A x^{2} \pm B x+C\right)=f(x) \quad \& \\
-2 \sqrt{C A}<B<+2 \sqrt{C A}
\end{gathered}
$$

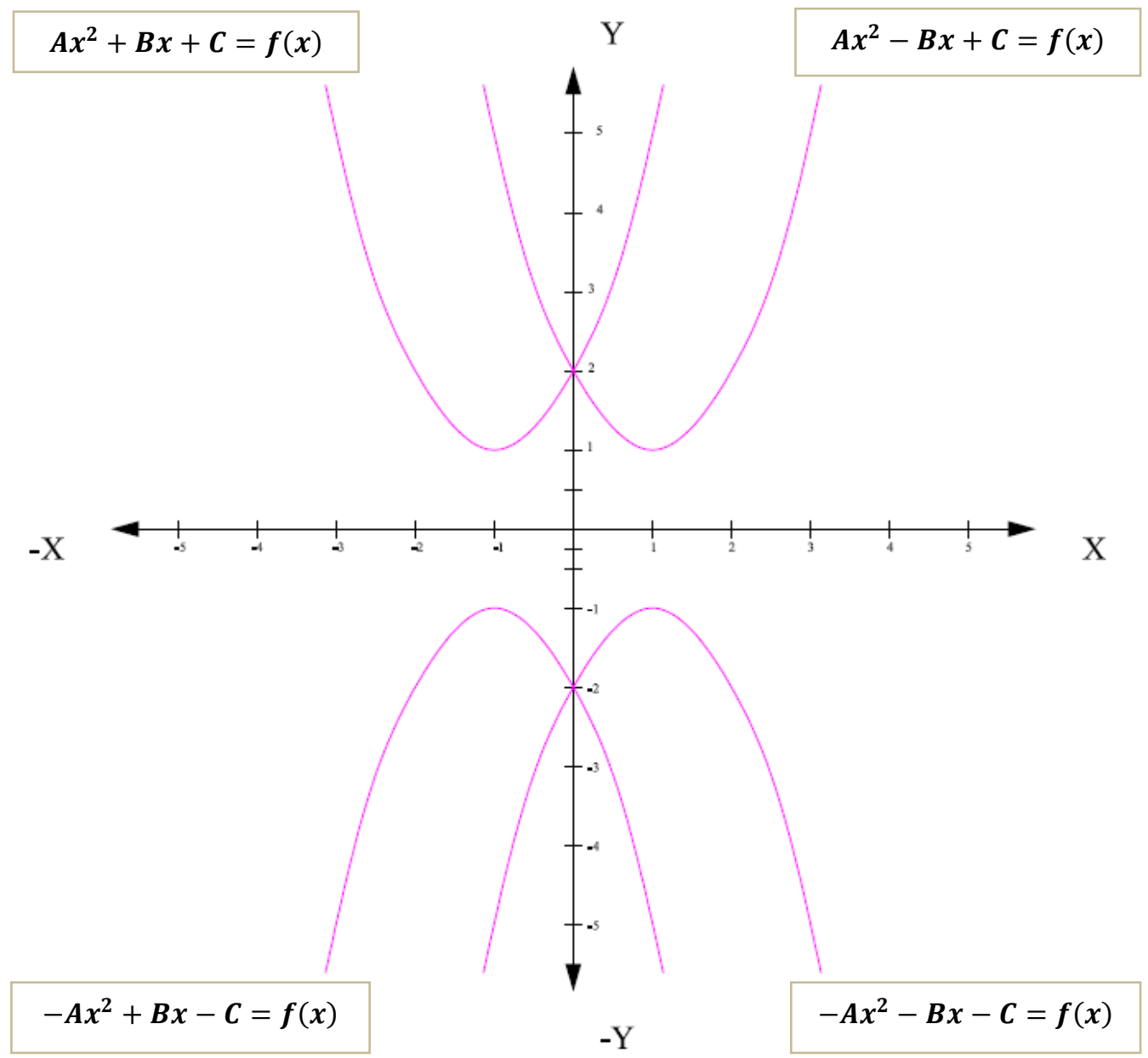

Fig (7)

From the visual inspection of these four types of Quadratic Functions we can see that even if we extend the Quadratic Equations to infinity it will never intersect the $X$ axis and will keep moving away from it. It is only these four cases of Quadratic Equations that will give complex solutions to its Quadratic Equations i.e. they have Complex Roots 